\title{
First results from three years of GNSS Interference Monitoring from Low Earth Orbit
}

\author{
Matthew J. Murrian*, Lakshay Narula ${ }^{\dagger}$, Peter A. Iannucci*, Scott Budzien ${ }^{\ddagger}$, \\ Brady W. O’Hanlon ${ }^{\S}$, Mark L. Psiaki ${ }^{\Uparrow}$, Todd E. Humphreys* \\ ${ }^{*}$ Department of Aerospace Engineering and Engineering Mechanics, The University of Texas at Austin \\ ${ }^{\dagger}$ Department of Electrical and Computer Engineering, The University of Texas at Austin \\ ${ }^{\ddagger}$ Naval Research Laboratory \\ $\S$ Cornell University \\ "Department of Aerospace and Ocean Engineering, Virginia Tech
}

\begin{abstract}
Observation of terrestrial GNSS interference (jamming and spoofing) from low-earth orbit (LEO) is a uniquely effective technique for characterizing the scope, strength, and structure of interference and for estimating transmitter locations. Such details are useful for situational awareness, interference deterrence, and for developing interference-hardened GNSS receivers. This paper presents the results of a three-year study of global interference, with emphasis on a particularly powerful interference source active in Syria since 2017. It then explores the implications of such interference for GNSS receiver operation and design.
\end{abstract}

Index Terms-GNSS interference; spoofing; emitter localization; Doppler positioning

\section{INTRODUCTION}

This paper presents the results of a three-year study of terrestrial GNSS interference as observed through a softwaredefined GNSS receiver operating since February 2017 on the International Space Station (ISS). The FOTON receiver, developed by The University of Texas at Austin (UT) and Cornell University, is part of a larger science experiment called GPS Radio Occultation and Ultraviolet Photometry-Colocated (GROUP-C), an unclassified experiment aboard the ISS that is part of the Space Test Program-Houston Payload 5 (STP-H5) payload. Serendipitous observations of GNSS interference in the occultation data are an important early result of GROUPC's scientific objective to characterize GPS signals in the LEO environment. This paper discusses the interference signals detected, their effects, and interference mitigation strategies for receivers deployed in LEO and terrestrial environments.

The FOTON receiver is a science-grade spaceborne dualfrequency (GPS L1 and L2) GNSS receiver [1]. Three levels of FOTON data are available for interference analysis: (1) raw 5.7 Msps intermediate frequency (IF) samples output by the FOTON front-end's analog-to-digital converter, (2) $100-\mathrm{Hz}$ data-modulation-wiped complex correlation products, and (3) 1-Hz standard GNSS observables (pseudorange, carrier phase, carrier-to-noise ratio $C / N_{0}$ ).

Although spaceborne GNSS sensors have been used for remote sensing via radio occultation [2] and reflectometry [3], there is little public literature exploring their use for

Correspondence: matthew.murrian@utexas.edu monitoring terrestrial GNSS interference. [4] characterized interference observed at a LEO satellite, and approximated the location for one source, but was mainly concerned with determining whether the interference had a detrimental impact on GPS-RO (radio occultation) meteorological products. The more recent survey of GNSS interference localization techniques in [5] makes no mention of single-receiver Dopplerbased localization, whether space-based or not.

General TDOA and FDOA interference localization has been extensively studied [6]-[10], and such techniques have been applied for terrestrial interference localization from geostationary orbit [11]-[13]. Application of T/FDOA for localization from LEO can be viewed as an extension of such demonstrations, with the lower-altitude orbits enabling localization of much weaker signals. Interference localization using a single satellite has been explored in [14], but only simulation results are presented, and these unrealistically assume perfecttone interference with a known and constant frequency.

This paper makes three primary contributions. First, it improves on the global survey technique in [4] by compensating for predictable $C / N_{0}$ variations in the detection test. Second, it presents the results of a three-year study of global GNSS interference, with emphasis on a powerful interference source active in Syria since 2017. Via Doppler positioning using the FOTON instrument on the ISS, an estimate of the transmitter's location is obtained whose horizontal errors are less than 1 $\mathrm{km}$ with $99 \%$ confidence based on reasonable clock and noise models. Such an accurate localization of a GNSS interference source from LEO is without precedent in the open literature. Third, this paper explores the implications of interference of the type generated by the source active in Syria for GNSS receiver operation and design.

A preliminary version of this paper was published in [15]. The current version focuses on the observed interference, extends the analysis period to June 2020, offers a more detailed analysis of localization accuracy, and includes a new section exploring implications for GNSS receivers.

\section{Single-SATellite TerRestrial Source GEOLOCATION}

As a prelude to the presentation of results from the observation campaign, this section introduces and analyzes the 
Doppler-based technique employed to estimate the location of the interference source operating in Syria.

Assuming a carrier can be extracted from an interference signal, single-satellite-based transmitter geolocation is possible from Doppler measurements alone [16], [17]. The analysis presented here emphasizes the effect of transmitter clock stability on geolocation accuracy.

Consider a static transmitter emitting a signal at the GPS L1 frequency as observed by a moving receiver. Let $\lambda$ be the signal wavelength in meters, $\hat{\boldsymbol{r}}$ the unit vector pointing from the transmitter to receiver, expressed in Earth-centeredEarth-fixed (ECEF) coordinates, $\boldsymbol{v}_{\mathrm{R}}$ the receiver velocity with respect to the ECEF frame and expressed in ECEF in $\mathrm{m} / \mathrm{s}$, and $\delta \dot{t}_{\mathrm{R}}$ the receiver clock frequency error in $\mathrm{s} / \mathrm{s}$, all at the time of signal receipt. Further, let $\delta \dot{t}_{\mathrm{T}}$ be the transmitter clock frequency error in s/s at the time of signal transmission, and $w$ be a zero-mean Gaussian error term that models thermal noise, ionospheric and tropospheric delay rates, and other minor effects, in Hz. Then the observed Doppler frequency in $\mathrm{Hz}$ at the receiver can be modeled as

$$
f_{\mathrm{D}}=-\hat{\boldsymbol{r}}^{T} \boldsymbol{v}_{\mathrm{R}} / \lambda-c\left[\delta \dot{t}_{\mathrm{R}}-\delta \dot{t}_{\mathrm{T}}\left(1-\delta \dot{t}_{\mathrm{R}}\right)\right] / \lambda+w
$$

where $c$ is the speed of light in $\mathrm{m} / \mathrm{s}$. It is assumed that $\boldsymbol{v}_{\mathrm{R}}$, $\delta \dot{t}_{\mathrm{R}}$, and the receiver position are known, e.g., via a GNSS receiver co-located with the transmitted signal receiver. The unknowns in (1) are transmitter position, which is embedded in $\hat{\boldsymbol{r}}$, and $\delta \dot{t}_{\mathrm{T}}$. The former is modeled as an unknown constant and the latter as a random walk process that evolves as

$$
\dot{\delta} t_{\mathrm{T}}\left(t_{k+1}\right)=\dot{\delta} t_{\mathrm{T}}\left(t_{k}\right)+v\left(t_{k}\right)
$$

Here, $v\left(t_{k}\right)$ is a discrete-time Gaussian random process with $\mathbb{E}\left[v\left(t_{k}\right)\right]=0$ and $\mathbb{E}\left[v\left(t_{k}\right) v\left(t_{j}\right)\right]=2 \pi^{2} h_{-2} \delta t \delta_{k, j}, \quad \forall k, j$, where $h_{-2}$ is the first parameter of the standard clock model based on the fractional frequency error power spectrum, as given in [18, Chap. 8]; $\delta t=t_{k+1}-t_{k}$ is the uniform sampling interval; and $\delta_{k, j}$ is the Kronecker delta.

A transmitter could introduce any level of complexity to carrier-phase frequency behavior; e.g., frequency modulation, frequency hopping, etc. Such behaviors, if not discovered and appropriately modeled, would confound single-pass geolocation efforts. Here, it is assumed that a nominally-constant carrier frequency is intended by the transmitter and that it is operating in steady-state conditions. In fact, it will be assumed that $h_{-2}$ is sufficiently small that $\dot{\delta} t_{\mathrm{T}}$ can be modeled as constant over a short (e.g., 60-second) data capture interval.

Based on the above Doppler measurement model, a batch maximum likelihood estimator [19] can be developed to estimate the unknown transmitter position and a constant value for $\dot{\delta} t_{\mathrm{T}}$ from a collection of single-pass Doppler measurements. If Doppler measurements from multiple satellite passes are available, these can be combined for single-batch estimation provided that a new value of $\dot{\delta} t_{\mathrm{T}}$ is estimated for each pass. In other words, $\dot{\delta} t_{\mathrm{T}}$ is viewed as constant over each short capture interval but variable from capture to capture.

When $\dot{\delta} t_{\mathrm{T}}$ is modeled as constant over a capture interval, actual transmitter clock instability gives rise to Doppler measurement errors. The impact of such errors on geolocation accuracy was analyzed via Monte Carlo simulation for three levels of transmitter clock quality, from a temperaturecompensated crystal oscillator (TCXO) to a laboratory-grade oven-controlled crystal oscillator (OCXO). Simulation parameters were based on the real-world interference capture discussed in the next section: the true transmitter location was simulated to be $35.4 \mathrm{~N}$ latitude, $35.95 \mathrm{E}$ longitude, $48 \mathrm{~m}$ altitude; the receiver trajectory was taken from the ISS orbit during the first 60 seconds of the capture interval on day 144 of 2018 (resulting in $441.65 \mathrm{~km}$ of total receiver displacement); and the measurement rate was $20 \mathrm{~Hz}$. First, an error-free Doppler time history was generated based on this scenario. Then, for each instance of the Monte Carlo simulation, an independent realization of a Doppler error random process consistent with the clock model being analyzed was generated and added to the error-free Doppler. Doppler error was modeled as a random walk process consistent with (2). These models assume a smooth compensation for temperature control, such as is common for TCXOs used in GNSS receivers. Additionally, $h_{-2}$ is assumed to dominate frequency stability over each short capture interval.

1000 Monte Carlo trials were conducted for each of the three clock quality levels. Transmitter horizontal location estimation errors were observed to be zero-mean and apparently Gaussian, and they were consistent with the formal error ellipses of the associated linear least-squares estimator. To determine whether 1000 trials were sufficient for a confident error analysis, subgroups of 250 trials were randomly selected from the 1000 trials and each of their geolocation error ellipses were calculated. Those subgroup samples were observed to deviate less than $\pm 10 \%$ from the population mean with $99 \%$ empirical confidence. For example, $99 \%$ of the subgroups for the TCXO simulation had geolocation error ellipse estimates between $6900 \pm 660$ meters, semi-major axis, and $690 \pm 71$ meters, semi-minor axis. Out of $1 \times 10^{5}$ subgroup samples drawn for each clock quality level, none were observed that deviated more than $17 \%$ from the population mean. Thus, 1000-trial-based error ellipses for each clock quality level given in Table I can be assumed to be no more than $15 \%$ smaller, on either axis, than the error ellipses that would be produced in the limit of an infinite number of trials.

TABLE I: Marginal contribution of transmitter frequency instability to a single-pass geolocation error ellipse. The size of the $95 \%$ horizontal geolocation error ellipse, in meters, is characterized by the semi-major $(a)$ and semi-minor $(b)$ axes.

\begin{tabular}{lcrl}
\hline Clock Quality & $h_{-2}$ & $a(\mathrm{~m})$ & $b(\mathrm{~m})$ \\
\hline TCXO & $3 \times 10^{-21}$ & 6900 & 690 \\
Low-quality OCXO & $3 \times 10^{-23}$ & 720 & 72 \\
OCXO & $3 \times 10^{-25}$ & 67 & 7.4 \\
\hline
\end{tabular}

Table I shows that marginal contribution of transmitter frequency instability to single-pass geolocation error grows precipitously with reduced transmitter clock quality. These results suggest that single-pass geolocation of a TCXO-based transmitter is marginal at best, and could be even worse if the $h_{-2}$ values for TCXOs in Table I are optimistic. On the other hand, if the transmitter is driven by an OCXO-quality 
clock, then clock instability contributes less than 720 meters (low-quality OCXO) or 67 meters (standard-quality OCXO).

The error ellipse characterized by $a$ and $b$ is highly eccentric, with semi-minor axis oriented in the direction of satellite motion: e.g., if the satellite is moving west to east then transmitter location will be best resolved in that direction. It follows that additional satellite passes provide the most benefit when, relative to the transmitter location, they are geometrically dissimilar to previous passes.

\section{ANALYSIS OF INTERFERENCE FROM SYRIA}

This section presents an in-depth analysis of a particular interference source active on the east coast of the Mediterranean Sea during the period of this paper's study, which spans from March 2017 to June 2020. The analysis illustrates the techniques that can be applied generally to study terrestrial GNSS interference sources using signals collected in LEO.

Recording raw IF data in LEO and relaying these to the ground for processing is an especially flexible approach well suited to studying new or poorly-understood interference. For the case presented here, the FOTON receiver captured 1minute intervals of raw 5.7-Msps two-bit-quantized IF samples at the GPS L1 (1575.42 MHz) and the GPS L2 (1227.6 MHz) frequencies. These data were packaged and downlinked via NASA's communications backbone. Ground processing using the latest version of UT's software-defined GNSS receiver [20] enabled analysis and tracking of all radio frequency signals near GPS L1 and L2.

The following observations are based on signals captured on three days in the first half of 2018 along the ground tracks shown in Fig. 1.

\section{A. Overview}

Strong interference is present in both the L1 and L2 bands, but the nature of the interference is markedly different between the two bands. At L2, the interference is narrowband, whereas at L1 it is a wideband spread-spectrum signal. The L1 interference is a composite of individual signals with a common carrier centered near GPS L1 but each having a unique GPS L1 C/A pseudo-random number (PRN) spreading code. Such interference can be categorized as matched-code GNSS interference [21], [22]. Signals corresponding to almost all GPS L1 C/A PRN codes from 1 to 32 have been detected. When tracked by the UT software-defined GNSS receiver, all false signals exhibit $C / N_{0}$ values greater than $40 \mathrm{~dB}-\mathrm{Hz}$. No discernible navigation data are modulated on the false GPS L1 signals. Moreover, the false signals are not clean simulated GPS L1 C/A signals: they exhibit unexplained fading and spectral characteristics. No false Galileo BOC $(1,1)$ signals were detected in the L1 band.

The lack of navigation bit modulation renders the signals ineffective at spoofing, but matched-code interference is a particularly potent form of jamming [21]. Why different techniques were used at L1 and L2 is unknown.

While some authentic GPS L1 C/A signals in the data are effectively jammed, the majority of authentic signals are still trackable owing to sufficient separation of corresponding false

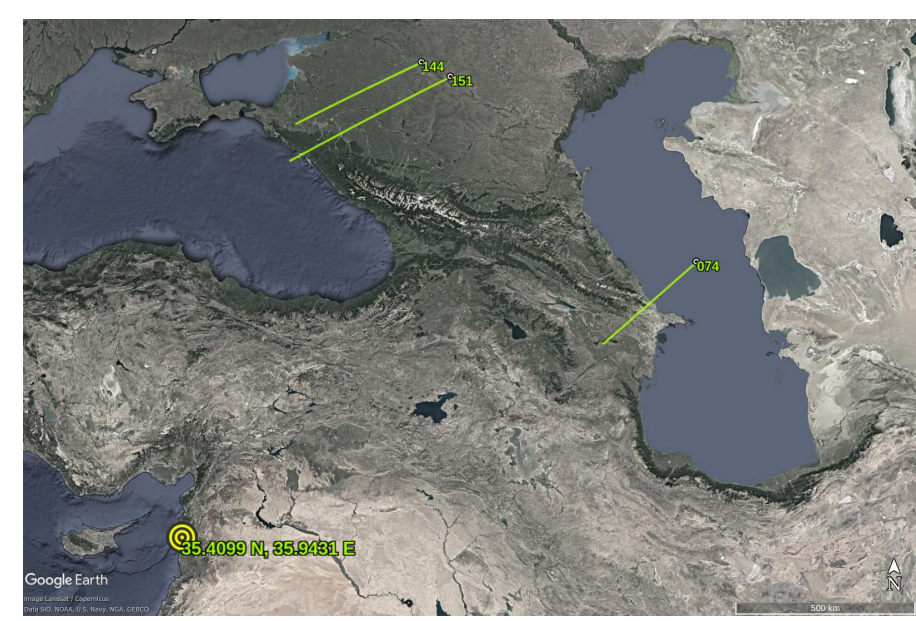

Fig. 1: Ground tracks for interference-affected captures on days 74, 144, and 151 of 2018. Each capture spans approximately 60 seconds. The estimated transmitter location is marked on the west coast of Syria.

and authentic signals in code-Doppler space. Thus, a correct receiver navigation solution can still be formed despite the interference.

\section{B. Power Spectral Characteristics}

Figs. 2 and 3 illustrate the captured signals' spectral characteristics. The spectra of narrowband interference near L2 are simple and remain similar across all three days, but the wideband interference at L1 is more complex and variable. It is clear from the left column of Fig. 2 that the matchedcode interference is cluttered by other components. Were it generated by a high-quality signal simulator, L1 interference would tend to be smooth like the authentic signals underlying the spectrum shown in the lower left panel. Instead, it appears to be an amalgam of components. Fig. 3 reveals that the rounded prominence in the L1 Day 144 panel exhibits oscillatory behavior with a 5-second period. Whether such variations are deliberate or are caused by transmitter idiosyncrasies is unknown.

\section{Baseband Signal Characteristics}

Fig. 4 shows time histories of 10-ms-accumulated complex correlation products from a false (top panel) and two authentic (bottom two panels) GPS L1 C/A signals present in the captured L1 band. The false signal's empirical $C / N_{0}$ value is $42.5 \mathrm{~dB}-\mathrm{Hz}$ on average, but the signal is highly irregular, manifesting both gradual and sudden fading. The gradual fading may be a result of scintillation as the signal passes upward through the lower ionosphere [24], but the sudden fading, highlighted in the inset of the top panel, is unnatural and likely originates at the transmitter.

\section{Source Geolocation}

The presence of a trackable carrier signal after despreading (cf. top panel of Fig. 4) enables geolocation of the interference 

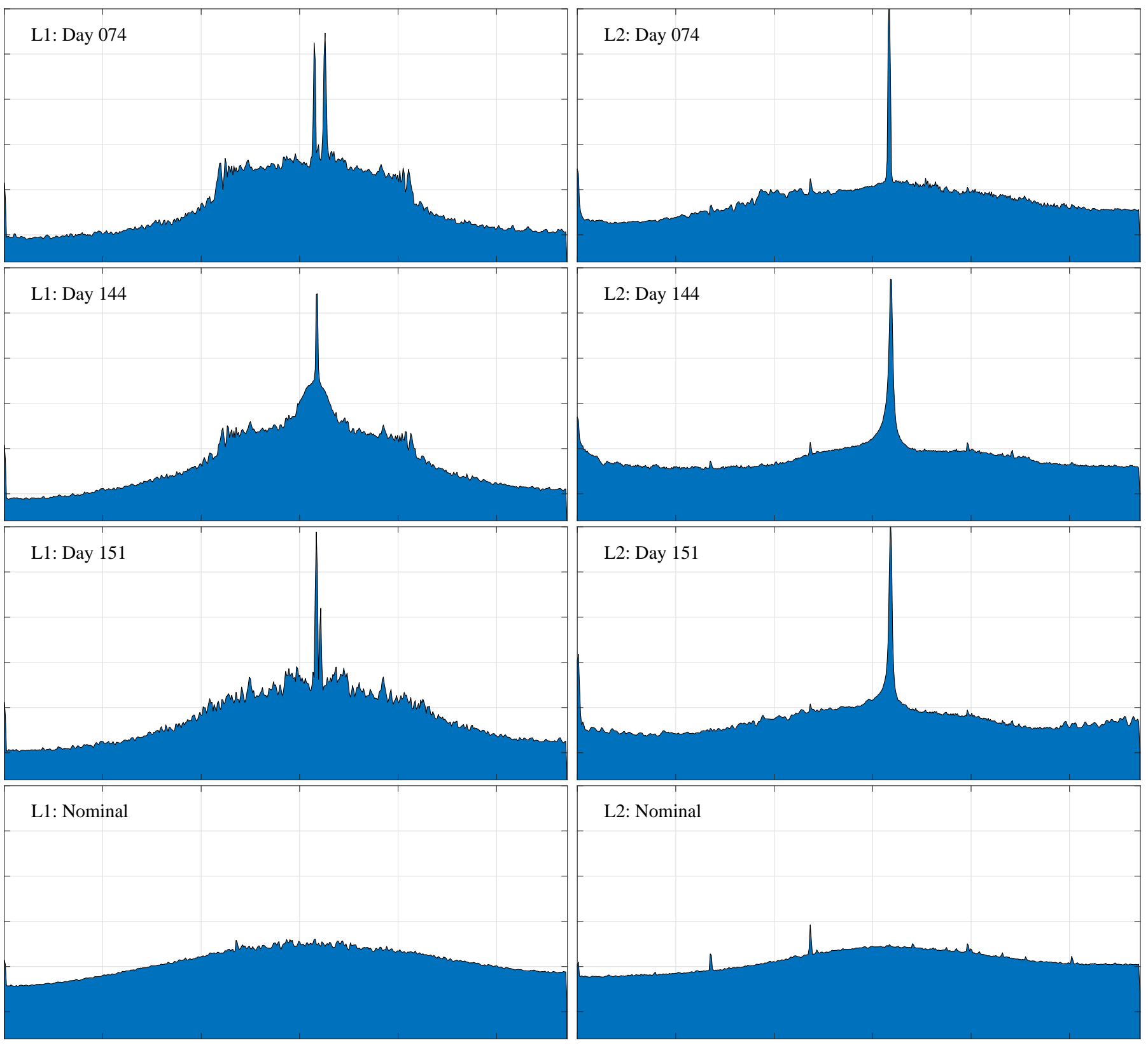

Fig. 2: Power spectra centered near the GPS L1 (left column) and L2 (right column) frequencies from interference-affected data captured on days 74, 144, and 151 of 2018 (top three rows), and from nominal data captured on day 158 of 2018 (bottom row). The frequency span is approximately $3 \mathrm{MHz}$ wide, scaled linearly with $0.5 \mathrm{MHz}$ divisions. All ordinate axes are in $\mathrm{dB}$ and scaled equivalently for ease of comparison. Spectra are estimated by Welch's method [23] from 1-second data intervals with a 5.6-kHz frequency resolution.

source as described in Section II. A receiver navigation solution was first estimated on days 74, 144, and 151 of 2018 using an Extended Kalman Filter (EKF) drawing in pseudorange and Doppler measurements extracted from the authentic GPS L1 C/A, GPS L2C, and Galileo E1 signals. Propagation of the receiver state estimate between measurement updates was based on a nearly-constant acceleration dynamics model. Time histories of the quantities $\boldsymbol{v}_{\mathrm{R}}, \delta \dot{t}_{\mathrm{R}}$, and the receiver position component of $\hat{\boldsymbol{r}}$ were then extracted from the EKF's state estimate and treated as known for purposes of source geolocation.
A batch estimator for interference source position and clock frequency bias was formulated as described in Section II. It was assumed that the interference observed on all three days originated from the same stationary transmitter, which allowed multiple days of Doppler measurements, collected on nonrepeating ground-tracks, to be combined to form a tightlyconstrained estimate. If these assumptions were false, large post-fit measurement residuals could be expected to manifest, which was not the case. Consistent with the assumption of a stationary transmitter, transmitter altitude was assumed to be near ground-level and was included as a pseudo-measurement. 


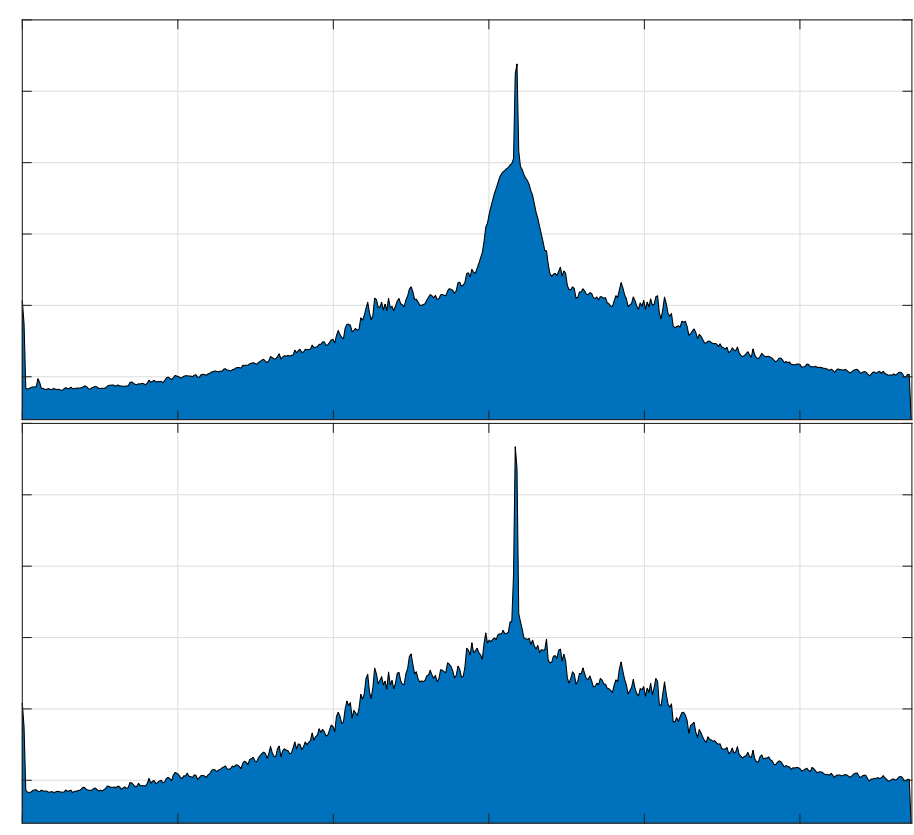

Fig. 3: Power spectra near L1 for the day 144 capture showing maximum (top) and minimum (bottom) phases of the waxing and waning wideband $(\sim 0.25 \mathrm{MHz})$ central interference prominence. The prominence oscillates with a period of approximately 5 seconds. The L1: Day 144 plot in Fig. 2 catches the prominence waning two seconds after the maximum shown in the top plot above.

A constant transmitter clock frequency error $\delta \dot{t}_{\mathrm{T}}$ was assumed to apply during each capture, but a new value of $\delta \dot{t}_{\mathrm{T}}$ was estimated for each of the three captures. Comparing the batch-estimator-produced estimates of $\delta \dot{t}_{\mathrm{T}}$ for days 74 and 144 revealed a two-sample transmitter clock frequency stability of approximately $\sigma_{y}(2, T, \tau)=6.85 \times 10^{-9}$ at a sampling interval $T$ of 70 days and an observation time (averaging interval) $\tau$ of approximately 50 seconds. The $B_{2}$ bias function [25] was used to convert this two-sample deviation to an Allan deviation, where $B_{2}(r, \mu)=1.8144 \times 10^{5}$ for $r=T / \tau$ and $\mu=1$, which assumes $h_{-2}$ is the dominant spectral component. This yielded an equivalent Allan deviation for $\tau=50$ seconds of $\sigma_{y}(2, \tau, \tau)=1.6 \times 10^{-11}$, which is consistent with a standardquality OCXO [26].

Thus, given the results of Table I, treating $\delta \dot{t}_{\mathrm{T}}$ as constant over each 60-second capture can be conservatively expected to introduce $95 \%$ errors smaller than 720 meters (that corresponding to a low-quality OCXO) in single-pass geolocation. A Monte-Carlo simulation like the one that produced the Table I data but for the combined three days of collection showed that, assuming independence in the clock frequency errors between passes, and conservatively assuming a low-quality OCXO, this error source can be expected to contribute $95 \%$ errors below 230 meters in the combined 3-day solution.

It is worth noting that, because $\delta \dot{t}_{\mathrm{R}}$ and $\delta \dot{t}_{\mathrm{T}}$ enter equivalently into the Doppler measurement model (1), and because no prior knowledge of these parameters is assumed in the batch maximum-likelihood estimator, an error in the EKF's estimate of $\delta \dot{t}_{\mathrm{R}}$ will directly manifest in the batch-estimator-produced
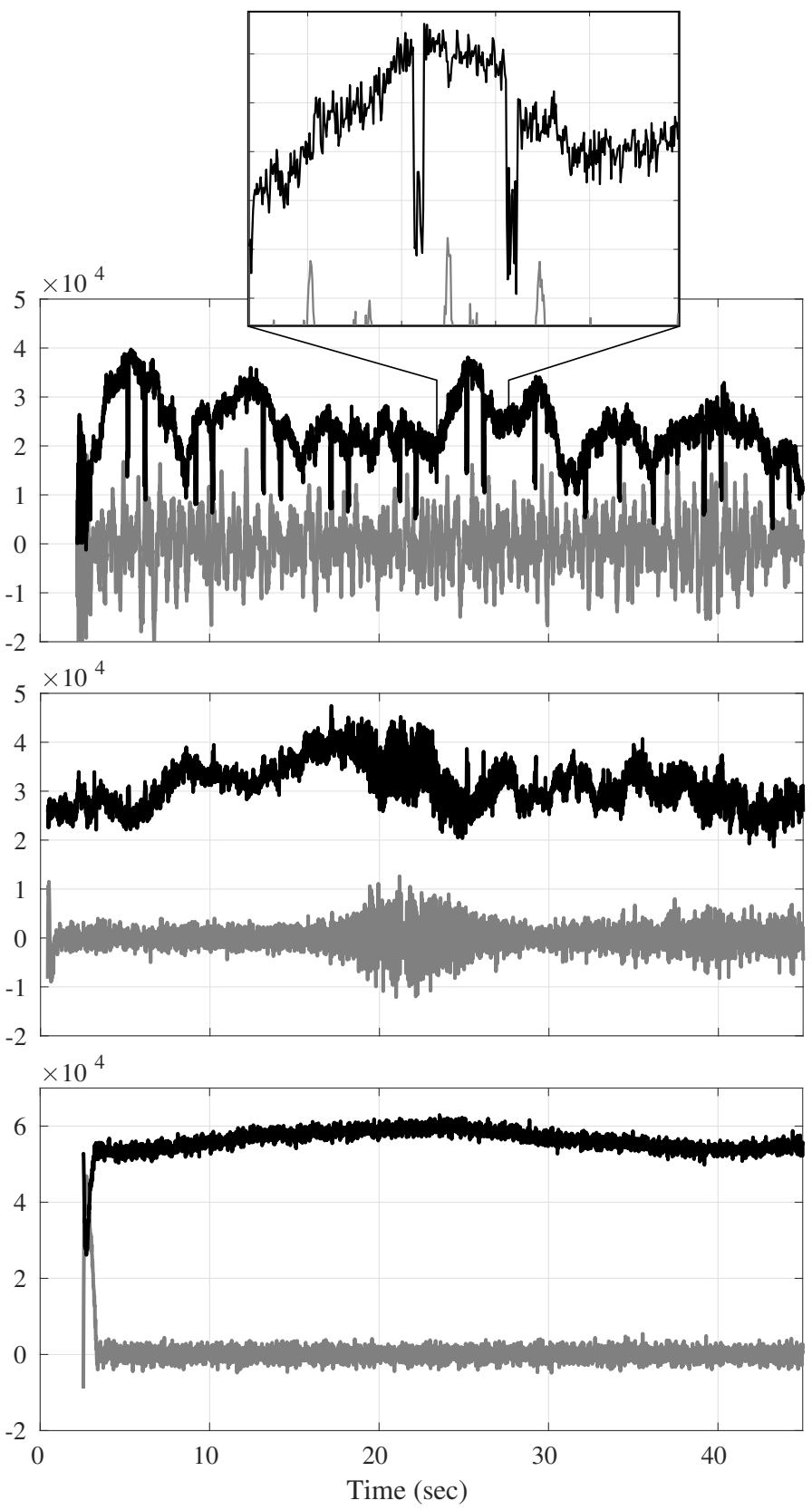

Fig. 4: In-phase (black) and quadrature (gray) 10-ms accumulation time histories for the strongest false signal from the day 74 capture (top), the strongest authentic signal from the day 74 capture (middle), and the strongest signal from the day 158 nominal capture (bottom). Data wipe-off was implemented in the middle and bottom figures. The inset on the top panel shows an amplified view of two sudden amplitude fades in the received false signal. The maximum carrier-to-noise ratio $C / N_{0}$ over the intervals shown are, from the top, $42.5,46.8$, and $52.5 \mathrm{~dB}-\mathrm{Hz}$.

estimate of $\delta \dot{t}_{\mathrm{T}}$ for each capture. However, examination of the the EKF's error covariance revealed that its estimate of $\delta \dot{t}_{\mathrm{R}}$ was good to better than $7 \times 10^{-10}(1-\sigma)$ for the day 74 and 144 captures. Thus, receiver-side errors are likely small enough that $\sigma_{y}(2, \tau, \tau)=1.6 \times 10^{-11}$ remains an accurate 

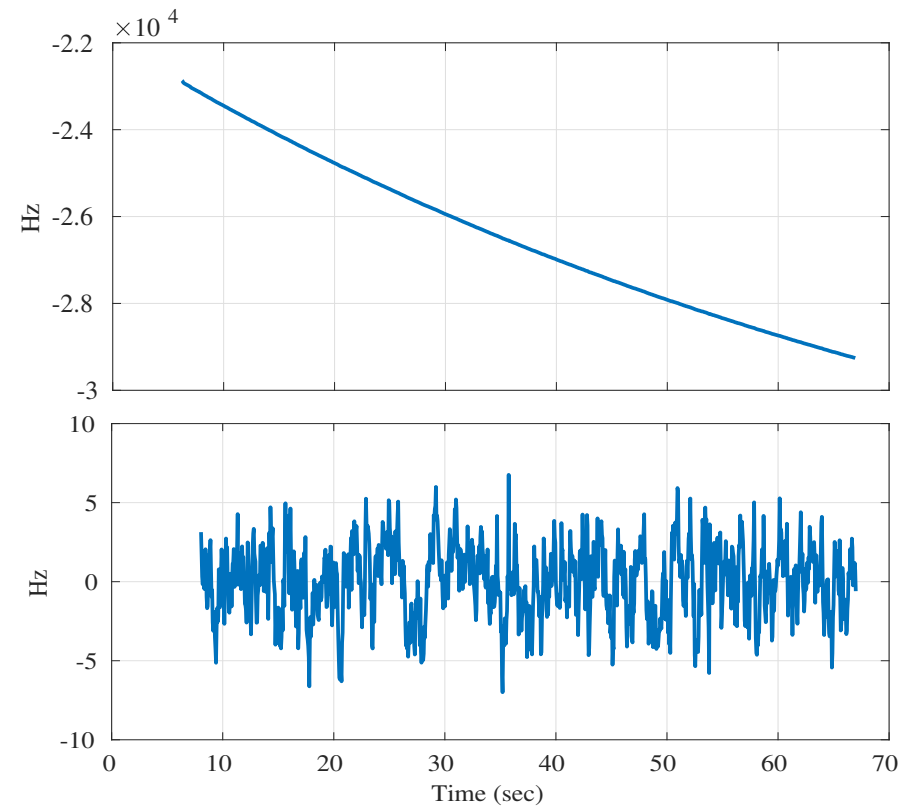

Fig. 5: Top: Doppler time history corresponding to the false PRN 10 signal from the day 144 capture. Bottom: Post-fit residuals of the Doppler time history assuming the estimated transmitter location and clock rate offset. The standard deviation of the post-fit residuals is $2.3 \mathrm{~Hz}$.

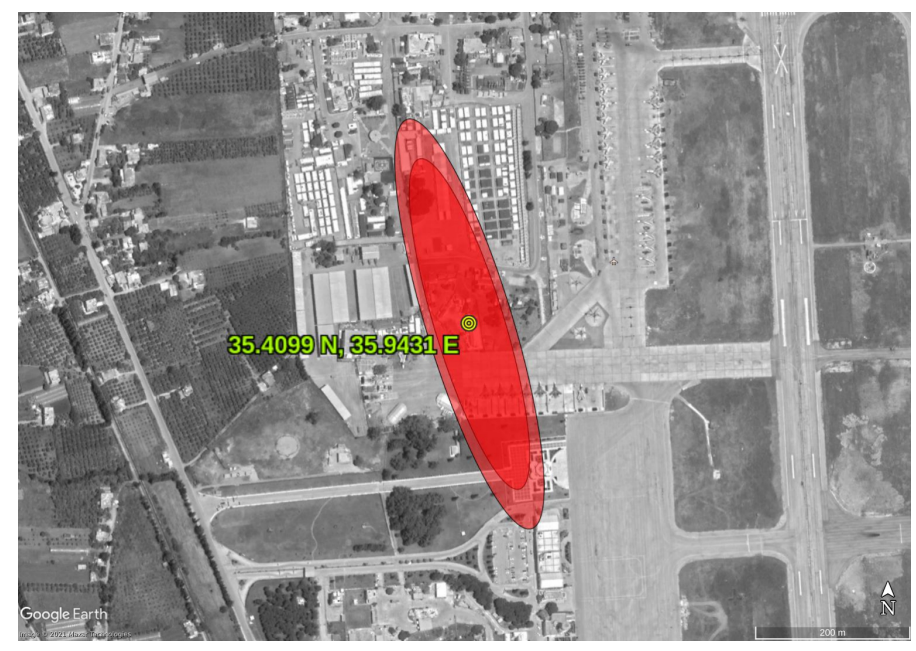

Fig. 6: Estimated transmitter location overlaid on formalerror $95 \%$ and $99 \%$ horizontal error ellipses. The location is coincident with an airbase on the coast of Syria. The semimajor axis of the $95 \%$ ellipse is 220 meters.

assessment of the transmitter clock stability.

Fig. 5 shows time histories of Doppler and post-fit residuals for false PRN 10 collected on day 144. The standard deviation of the post-fit residuals is $2.3 \mathrm{~Hz}$, indicating that the measurement model in (1), and the assumption of a constant $\delta \dot{t}_{\mathrm{T}}$ over each capture, are reasonably accurate. Fig. 6 shows the estimated position of the interference source. The horizontal error ellipses, which indicate a solution better than 220 meters (95\%), are formal error ellipses assuming (1) constant $\delta \dot{t}_{\mathrm{T}}$ over each capture, (2) a standard deviation of $5 \mathrm{~m}$ for the transmitter altitude constraint, and (3) a standard deviation between 2.3 and $2.5 \mathrm{~Hz}$ (depending on the empirical post-fit residuals for each capture) for the measurement error $w$ from (1). Assuming an OCXO-quality clock in the transmitter, the error caused by modeling a constant $\delta \dot{t}_{\mathrm{T}}$ is small compared to these formal error ellipses. While the true location is not known, the geolocation solution based on the model plausibly coincides with a Russian-operated air base in Syria.

\section{E. Transmitter Power}

In the presence of interference, $C / N_{0}$ actually measures the carrier-to-interference-and-noise ratio, CINR. By analyzing the authentic signal CINR values in the captured data one can infer the transmitted power in the direction toward ISS of the emitter located in Syria. The data presented here are for the day 74 capture. The average decrease in CINR observed at the ISS when $1340 \mathrm{~km}$ from the source was approximately $6 \mathrm{~dB}$. One may assume the interference acts as multi-access interference, whose spectral density is $I_{0}=(2 / 3) P_{\mathrm{I}} T_{C}$ [21], where $P_{\mathrm{I}}$ is the received interference power and $T_{C}=1023^{-1}$ $\mathrm{ms}$ is the GPS L1 C/A spreading code chip interval. Then, assuming $N_{0}=-204 \mathrm{dBW} / \mathrm{Hz}$, a drop in CINR by $6 \mathrm{~dB}$ implies $P_{\mathrm{I}}=-137 \mathrm{dBW}$. Let $P_{\mathrm{S}}=P_{\mathrm{I}}-G_{r}+L$, and assume path loss $L=159 \mathrm{~dB}$, consistent with a stand-off distance of $1340 \mathrm{~km}$, and receiver antenna gain $G_{r}=3 \mathrm{~dB}$. It follows that the transmitter power of the interference source in the direction toward the ISS during the day 74 capture is $P_{\mathrm{S}}=19$ $\mathrm{dBW}$, or $79 \mathrm{~W}$. If the transmitter is focused on ground based targets, then it is possible that the gain pattern is toroidal. The elevation angle of the ISS as seen from the transmitter is low during this period (varying between 8 and 13.5 degrees) and may have been near the maximum of a toroidal gain pattern.

\section{Global Interference Survey Via RECEIVER-REPORTED CINR}

The raw IF data captures from the ISS FOTON receiver enable detailed monitoring of GNSS interference signals and their structure, but such captures are infrequent and limited to short 1-minute intervals. By contrast, the 1-Hz standard GNSS observables and $100-\mathrm{Hz}$ data-wiped complex correlation products have been logged nearly continuously since early 2017 . These data facilitate a world-wide survey of strong GNSS interference.

\section{A. Calculation of Receiver-Reported CINR}

Receiver-reported CINR is calculated as

$$
\mathrm{CINR}=\left(\frac{\mathbb{E}\left[I^{2}+Q^{2}\right]}{2 \sigma_{I Q}^{2}}-1\right) \frac{1}{T_{a}}
$$

where the expectation $\mathbb{E}\left[I^{2}+Q^{2}\right]$ is estimated by moving average using a Euler approximation to a standard low-pass filter

$\mathbb{E}\left[I^{2}+Q^{2}\right]_{k}=\mathbb{E}\left[I^{2}+Q^{2}\right]_{k-1}+K\left(I_{k}^{2}+Q_{k}^{2}-\mathbb{E}\left[I^{2}+Q^{2}\right]_{k-1}\right)$

with subscripts $k$ and $k-1$ indicating the current and previous accumulation interval. The gain parameter $K=\frac{T_{a}}{\tau}$ with 
accumulation interval $T_{a}=10 \mathrm{msec}$ and filter time constant $\tau=0.5 . I_{k}$ and $Q_{k}$ are the in-phase and quadrature prompt correlation products for the current accumulation interval.

The receiver noise floor, $2 \sigma_{I Q}^{2}$, can be derived analytically for a 2-bit quantizing RF front-end and a software-defined GNSS receiver based on the quantization models of both the $\mathrm{RF}$ front-end and receiver local carrier replica. It can be shown that

$$
2 \sigma_{I Q}^{2}=2 N\left(a_{0}^{2} p_{a 0}+a_{1}^{2} p_{a 1}\right)\left(b_{0}^{2} p_{b 0}+b_{1}^{2} p_{b 1}\right)
$$

where $N$ is the number of samples per accumulation interval, $a_{0}, a_{1}, b_{0}$, and $b_{1}$ are the low and high quantization values of the RF front-end and local carrier replica, respectively, and $p_{a 0}, p_{a 1}, p_{b 0}$, and $p_{b 1}$ are their associated probabilities. In practice, $p_{a 0}$ and $p_{a 1}$ depend on the implementation of the automatic gain control (AGC) in the RF front end, and $p_{b 0}$ and $p_{b 1}$ are selected by the receiver designer, e.g., to minimize quantization distortion. The following values are applicable to the FOTON receiver

$$
\begin{aligned}
a_{0} & =1, a_{1}=3, b_{0}=1, b_{1}=3 \\
p_{a 0} & =0.68269, p_{a 1}=0.31731, p_{b 0}=0.38418, p_{b 1}=0.61582 \\
N & =5714.286
\end{aligned}
$$

which yield a noise floor of $2 \sigma_{I Q}^{2}=239669$ front-end units.

\section{B. Methodology}

The carrier power $C$ of an authentic signal can be modeled as a function $C\left(j, f, r_{s r}, z_{s}, z_{r}\right)$, where $j$ is the GNSS satellite identifier (SV ID), $f$ is the frequency band (L1 or L2), $r_{s r}$ is the range between the GNSS satellite antenna and the ISS FOTON antenna, $z_{s}$ is the angle between the satellite boresight direction and the direction to the ISS antenna (i.e., the satellite antenna off-boresight angle), and $z_{r}$ is the angle between the ISS antenna boresight direction and the direction to the satellite (receiver antenna off-boresight angle). A hypothesis test based on the receiver-reported CINR can be designed to detect whether $\left(H_{1}\right)$ or not $\left(H_{0}\right)$ the receiver is experiencing interference. Under a given $P_{\mathrm{F}}$, this requires that the statistics $\mathbb{E}\left[l \mid H_{0}\right]$ and $\operatorname{Var}\left(l \mid H_{0}\right)$ be known. To obtain these statistics, this section assumes the receiver reports interference-free data (consistent with $H_{0}$ ) when the ISS is over deep ocean bodies.

To isolate the variations in reported CINR due to interference, the data are first pre-processed to eliminate the predictable sources of carrier power variation. First, the dependence of $C$ on $r_{s r}$ is removed by compensating for the free space path loss:

$$
\hat{C}\left(j, f, z_{s}, z_{r}\right)=C\left(j, f, r_{s r}, z_{s}, z_{r}\right) \times\left(\frac{4 \pi r_{s r} f}{c}\right)^{2}
$$

Modeling of interference-free $C / N_{0}$ is complicated by the ISS's local multipath environment. The ISS antenna is flanked by solar panels that move with respect to the FOTON antenna, causing a non-stationary signal obstruction and multipath environment. Nevertheless, an off-boresight angle window $z_{r} \in\left[0^{\circ}, 15^{\circ}\right]$ is known to be free of obstructions. Only the signals received in this window are considered for interference detection in this paper's analysis. Confining $z_{r}$ to this window

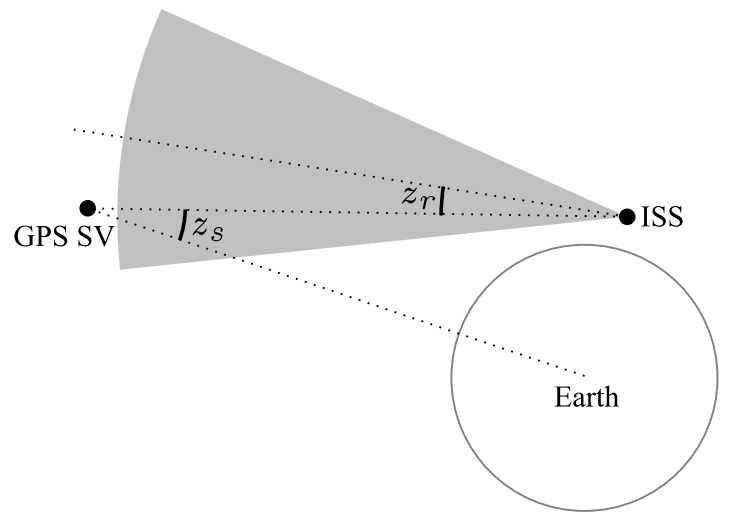

Fig. 7: For receiver off-boresight angle $z_{r} \leq 15^{\circ}$ (within the gray region), the satellite off-boresight angle $z_{s}$ is restricted between $14.2^{\circ} \leq z_{s} \leq 15.2^{\circ}$

restricts the geometry between GNSS satellites and the ISS such that $z_{s} \in\left[14.2^{\circ}, 15.2^{\circ}\right]$ (see Fig. 7). The GNSS antenna gain pattern can be assumed to be relatively constant over $\pm 0.5^{\circ}$. Thus, $\hat{C}\left(j, f, z_{s}, z_{r}\right)$ can be assumed independent of $z_{s}$.

The mean and variance of ISS-reported range-compensatedCINR values $\hat{C} / N_{0}$ collected over deep ocean regions are maintained as control data in a three-dimensional grid of SV ID $j$, frequency band $f$, and receiver off-boresight angle $z_{r}$. For a world-wide analysis of GNSS interference events, a hypothesis test is performed on the test statistic derived from $\hat{C} / N_{0}$ values that fall within $z_{r} \in\left[0^{\circ}, 15^{\circ}\right]$. The test is performed separately for the L1 and L2 bands since the interference characteristics are frequency dependent. If the reported test statistics falls below $\mathbb{E}\left[l \mid H_{0}\right]-3 \sqrt{\operatorname{Var}\left(l \mid H_{0}\right)}$, the receiver is declared to be under interference. This threshold respects a $P_{\mathrm{F}}$ of approximately $1.35 \times 10^{-3}$.

\section{Discussion of Results}

Fig. 8 shows the ratio of the number of potential interference events recorded at L1 (top panel) and L2 (bottom panel) to total number of hypothesis tests performed at each location for the foregoing detection threshold. As expected, a high ratio of potential interference events is reported for both L1 and L2 near Syria (marked with a red dot). Note that the interference "hotspot" appears to the east of the source because the ISS orbit is prograde and the FOTON antenna points in the anti-velocity direction. In other words, the FOTON antenna is exposed to interference only after the ISS passes eastward over an emitter's location.

The high values of the statistic for both L1 and L2 east of Syria indicate that the interference activity in Syria has been persistent over nearly the full interval considered in this paper, from March 2017 to June 2020. A monthly analysis (not shown) revealed that the source has been transmitting at L2 since no later than March 2017. It began transmitting weak interference at L1 during the second half of 2017, then much stronger interference at L1 during the first quarter of 2018. The interference at L1 and L2 was ongoing in June 2020. 


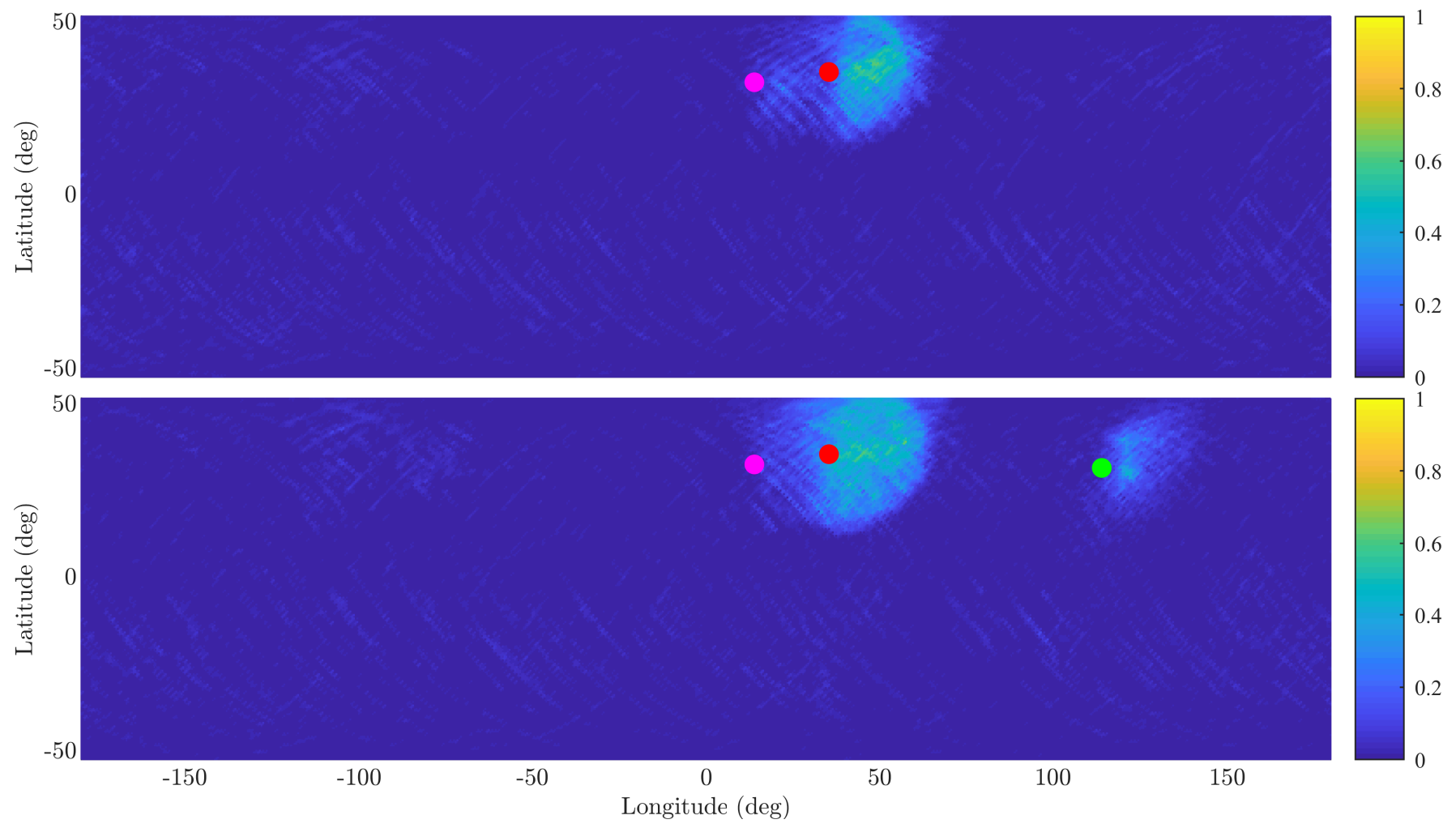

Fig. 8: Ratio of number of potential GPS L1 (top panel) and L2 (bottom panel) interference events recorded to total number of hypothesis tests performed at each location on the map for the full span of data considered in this paper, from March 2017 to June 2020. The red dots indicate the estimated origin of the interference from Syria based on raw IF recordings. Another hotspot of interference is apparent to the west of the Syrian location. The magenta dots denote the approximate location of GNSS interference reports in the Libyan region [27]. In addition to the interference over the Syrian and Libyan regions, strong L2 interference over mainland China is observed. The green dot at $\left(32^{\circ} \mathrm{N}, 114^{\circ} \mathrm{E}\right)$ indicates a hypothesized interference source location based on the shape and location of the observed hotspot.

An additional hotspot is present to the west of the Syrian location. This hotspot, which emerged in the second half of 2019 , is consistent with reports of GNSS interference in the Libyan region [27]. The magenta dots in Fig. 8 denote the approximate location of the area in which interference has been documented $\left(33^{\circ} \mathrm{N}, 14^{\circ} \mathrm{E}\right)$. Fig. 8 also reveals strong L2 interference over mainland China. This interference has been present since March 2017 at the latest and was ongoing in June 2020. The green dot in Fig. 8, marked at $\left(32^{\circ} \mathrm{N}, 114^{\circ}\right.$ $\mathrm{E})$, indicates a hypothesized interference source location based on the shape and location of the observed hotspot.

Note that the above method of counting potential interference events based on CINR degradation ignores cases where interference might lead to complete loss of track of some or all GPS signals. However, the data from the ISS shows that FOTON does not lose track of authentic GNSS signals even when flying by the strong interference source in Syria. In fact, the reported CINR over Syria is well above the weakest signal that FOTON is capable of tracking. As a result, it was concluded that in cases where FOTON seems to track few or no GPS signals, it is likely due to some abnormal behavior of the receiver, and not due to a potential interference event.

In addition to the global average analysis summarized in Fig. 8, it is instructive to examine the time history of receiver reported CINR as the ISS passes over an interference hotspot. Fig. 9 shows two such histories for signals within the admissible range of $z_{r}$ as the ISS goes over the strong interference regions in Syria (Fig. 9(a)) and China (Fig. 9(b)). Green and blue data points represent range-compensated CINR values for authentic L1 and L2 GNSS signals, respectively, above the applicable threshold, which depends on $i, f$, and $z_{r}$. Light red data points are the same data when below the applicable threshold. Both L1 and L2 signals are declared under interference in Fig. 9(a), whereas only L2 signals are declared under interference in Fig. 9(b). The brief dip in Fig. 9(b) prior to the major dip over China is caused by the interference originating in Syria. Gaps in the time histories indicate periods with no tracked signals in the admissible offboresight angle window.

\section{IMPLICATIONS FOR GNSS RECEIVERS}

The matched-code interference captured over Syria is intriguing. So far as this paper's authors are aware, no other GNSS interference captured from an operational (as opposed to experimental) source has exhibited the characteristics observed in the interference emanating from Syria. If the intent behind the signals transmitted at L1 is not spoofing but rather denial of GPS service, as might be inferred from the lack 


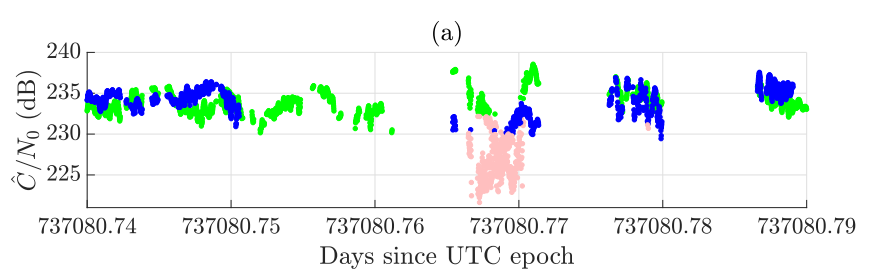

(b)

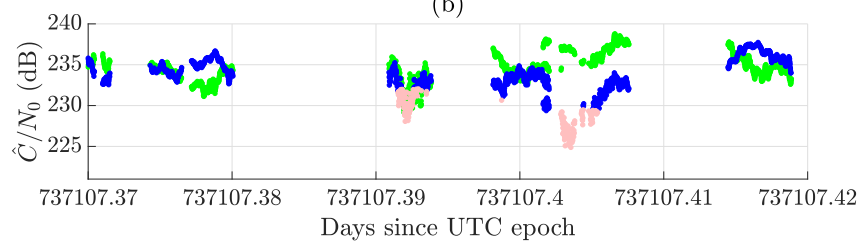

Fig. 9: Time histories of range-compensated receiver-reported CINR as the ISS flies over potential GPS interference zones over Syria and China.

of navigation data bit modulation, then it is unclear why an ensemble of signals, each one modulated by a separate GPS L1 C/A spreading code, was transmitted. The transmitter could just as well allocate its power to a single GPS L1 C/A spreading code, or any code with a similar spectral density. However, transmitting a multitude of spreading codes can be effective at disrupting cold-start acquisition of GPS L1 C/A signals, as explained below.

\section{A. Efficient Jamming}

The art of jamming is more sophisticated than merely emitting RF energy into a target band. An efficient jammer is one that effectively disrupts GNSS service in a given area of operations but does so with as little power as possible. Such frugality extends the life of battery-powered jammers, and makes jammers less conspicuous. The key to efficient jamming is avoiding wasteful allocation of signal power. Obviously, allocating power outside a target receiver's passband is wasteful because the interference is filtered out by the receiver's RF front-end. Less obviously, narrowband jamming applied directly in the passband is also wasteful. To understand this, consider the vector space of all possible input signals, and a partitioning into a subspace that contains the jamming signal and one that does not. If the jammer-occupied subspace is sparse with respect to the desired signal subspace, and if the receiver's front-end amplification and quantization are not saturated, then a technique can be developed to excise the jammer-occupied subspace with minimal degradation to the desired signals. For a narrowband jammer, the technique is notch filtering; for a pulsed jammer, the technique is pulse blanking [21].

An efficient jammer maximizes overlap with the desiredsignal subspace for a given power allocation. Jamming that is continuous in the time domain and white (spectrally flat) within the desired signal passband in the frequency domain is reasonably efficient because it extensively overlaps the desired signal subspace. Continuous-time matched-spectrum jamming is even more efficient: Instead of spreading the jamming power evenly across the passband, a matched-spectrum jammer shapes it for greater overlap with the desired signal subspace.
Consider a random binary spreading code with chip interval $T_{\mathrm{C}}$. Suppose a spectrally-flat jammer is designed to cover the spreading code's primary spectral lobe and first two side lobes, for a total frequency span of $4 / T_{\mathrm{C}} \mathrm{Hz}$. The noise power density that passes through the receiver's matched filter is $I_{0}=P_{\mathrm{I}} T_{\mathrm{C}} / 4$, where $P_{\mathrm{I}}$ is the interference power. By contrast, for a matched-spectrum jammer $I_{0}=(2 / 3) P_{\mathrm{I}} T_{\mathrm{C}}$ [21]. When $I_{0}$ is large enough that CINR $\approx C / I_{0}$, the matched-spectrum jammer is $4.3 \mathrm{~dB}$ more potent than the spectrally-flat jammer. What is more, the spectrally-flat jammer spanning $4 / T_{\mathrm{C}} \mathrm{Hz}$ can be excised by filtering in the frequency domain: even if the main lobe and adjacent two side lobes of the authentic signals are removed along with the jamming, the authentic signals are only attenuated by $13 \mathrm{~dB}$. The spectrally-flat jammer must spread its power even wider to avoid such excision by filtering, resulting in an even less favorable potency compared with matched-spectrum jamming. By contrast, a matched-spectrum jammer cannot be excised by filtering because its spectrum follows the $\operatorname{sinc}^{2}\left(f T_{\mathrm{C}}\right)$ envelope of the authentic binary-codemodulated signals. Thus, spectrum matching is a necessary condition for efficient jamming.

However, spectrum matching is not a sufficient condition for effective jamming. Consider a jammer emitting a carrier modulated only by a single publicly-known spreading code of arbitrary length. This signal is sparse with respect to the desired signal subspace. It can be excised by the receiver generating a local replica of the interference signal, aligning this replica's code phase, carrier phase, and amplitude with the interference signal, and subtracting the replica from the digitized output of the receiver's RF front end. Assuming sufficient front-end bit depth and amplifier linearity, this procedure can be extended to an arbitrary number of such interference signals, each with a known waveform; the technique is known as successive interference cancellation (SIC) [28].

Thus, an effective jammer will avoid predictable signals: a more sophisticated approach to spectrum matching is modulation of the carrier with a non-repeating spectrum-matching spreading code known only to the jammer. But this is only necessary when the target receiver is capable of SIC. If, for example, the receiver has no way of distinguishing authentic from interference signals, then it cannot apply SIC without also eliminating desired signals.

\section{B. The Cold Start Vulnerability}

It is instructive to consider the conditions under which a GNSS receiver is unable to distinguish between authentic and interference signals: (1) the authentic and interference signals are identical in all aspects of significance (modulation, code phase, carrier phase and frequency, amplitude), or (2) the authentic and interference signals are identical except in ways the target receiver is unable to exploit to distinguish them. In case (1), the interference is hardly a problem: it simply reinforces the authentic signals. Case (2) is more interesting. Let the term spoofing interference refer to matched-code interference with all additional modulation requisite to make the interference signal's structure and content identical to an authentic signal's. If a receiver is exposed to spoofing interference while already 
tracking enough authentic signals to form a navigation solution and when in possession of accurate satellite ephemerides, it can distinguish any authentic and interference signals that differ in code phase, carrier frequency, or amplitude. (It can additionally distinguish by carrier phase if performing precise carrier-based navigation.) Therefore, jamming a navigationlocked receiver with spoofing interference may be ineffective because the target receiver can apply SIC.

However, during a cold start, the target receiver's time and position are uncertain, and it lacks the ephemerides necessary to predict the code phase and Doppler of authentic signals even if its time and position were known. In this case the receiver is highly vulnerable to spoofing interference. Suppose a jammer generates a counterpart power-matched spoofing signal for each authentic GNSS signal available in an area of operations. Suppose further that the ensemble of spoofing signals is self-consistent with a location and time different from the target receiver's true location and time. On cold start, the receiver is jammed not in the traditional sense of being unable to acquire and track the authentic signals, but rather in the sense of being unable to confidently declare which of two plausible-looking navigation solutions is correct. If, under this circumstance, the receiver refuses to provide a navigation solution, the user is effectively denied GNSS service. If instead the receiver mistakenly provides the spoofed solution, the user could be exposed to hazardously misleading information.

This type of spoofing interference is highly efficient. Suppose the target receiver has a cold-start CINR acquisition threshold of $\eta \mathrm{dB}-\mathrm{Hz}$. Then traditional matched-spectrum jamming would require a jamming-to-authentic power ratio equal to

$$
\frac{P_{\mathrm{I}}}{C}=-\left[\eta+10 \log _{10}\left(\frac{2 T_{\mathrm{C}}}{3}\right)\right]
$$

which, for GPS L1 C/A signals and a typical $\eta=30 \mathrm{~dB}$ $\mathrm{Hz}$, amounts to $31.8 \mathrm{~dB}$. By contrast, jamming via singlecounterpart power-matched spoofing interference requires only $P_{\mathrm{I}} / C=0 \mathrm{~dB}$, which makes it more than 1500 times more efficient for denial of GNSS service at cold start.

\section{Discussion}

The interference captured over Syria causes traditional matched-spectrum jamming at close range, and is capable of disrupting cold-start acquisition far beyond this (along its lineof-sight). Indeed, it would be at least partially effective at preventing FOTON cold start even at the maximum line-ofsight range to the ISS, or approximately $1600 \mathrm{~km}$. However, the interference signals as broadcast have at least four flaws, any one of which could be exploited by receivers to distinguish them from authentic signals: (1) they lack navigation data modulation; (2) they are broadcast on a common and constant carrier frequency; (3) they share a common code phase alignment; (4) they include signals for (almost) all GPS PRNs. A receiver built to detect these anomalies could identify the imposter signals and eliminate them via SIC.

However, in general, spoofing interference is not so easily distinguished from authentic signals, and can be both effective and power-efficient at denying GNSS service on cold start. The best defense against spoofing interference intended to deny GNSS service remains an open problem.

\section{CONCLUSIONS}

Low-earth-orbiting instruments capable of receiving signals in GNSS bands are a powerful tool for characterizing GNSS interference emanating from terrestrial sources. Data from one such instrument, the FOTON software-defined GNSS receiver, which has been operational on the International Space Station since February 2017, reveal interesting patterns of GNSS interference from March 2017 to June 2020. Signals from a particularly powerful and persistent interference source active in Syria since 2017 were captured and characterized, and the source was geolocated to better than $1 \mathrm{~km}$. A global analysis revealed other interference hotspots around the globe in both the GPS L1 and L2 frequency bands. Matched-code interference such as emitted at the GPS L1 frequency by the jammer in Syria is power-efficient for jamming signal-locked GNSS receivers. GNSS receivers are particularly vulnerable to such interference during cold start.

\section{ACKNOWLEDGMENTS}

Work at The University of Texas has been supported in part by the National Science Foundation under Grant No. 1454474 (CAREER) and in part by in part by the U.S. Department of Transportation (USDOT) under Grant 69A3552047138 for the CARMEN University Transportation Center (UTC). Work at the Naval Research Laboratory was supported by the Chief of Naval Research. The STP-H5/GROUP-C experiment was integrated and flown under the direction of the Department of Defense Space Test Program.

\section{REFERENCES}

[1] E. G. Lightsey, T. E. Humphreys, J. A. Bhatti, A. J. Joplin, B. W O'Hanlon, and S. P. Powell, "Demonstration of a space capable miniature dual frequency GNSS receiver,' Navigation, vol. 61, no. 1, pp. 53-64, Mar. 2014.

[2] C. O. Ao, G. A. Hajj, T. K. Meehan, D. Dong, B. A. Iijima, A. J. Mannucci, and E. R. Kursinski, "Rising and setting GPS occultations by use of open-loop tracking," Journal of Geophysical Research, vol. 114, no. D4, Feb. 2009.

[3] S. Jin and A. Komjathy, "GNSS reflectometry and remote sensing: New objectives and results," Advances in Space Research, vol. 46, no. 2, pp. 111-117, July 2010.

[4] O. Isoz, S. A. Buehler, K. Kinch, M. Bonnedal, and D. M. Akos, "Interference from terrestrial sources and its impact on the GRAS GPS radio occultation receiver," Radio Science, vol. 49, no. 1, pp. 1-6, Jan. 2014.

[5] A. G. Dempster and E. Cetin, "Interference localization for satellite navigation systems," Proceedings of the IEEE, vol. 104, no. 6, pp. 1318 1326, June 2016.

[6] K. Ho and Y. Chan, "Geolocation of a known altitude object from TDOA and FDOA measurements," IEEE Transactions on Aerospace and Electronic Systems, vol. 33, no. 3, pp. 770-783, July 1997.

[7] T. Pattison and S. Chou, "Sensitivity analysis of dual-satellite geolocation," IEEE Transactions on Aerospace and Electronic Systems, vol. 36, no. 1 , pp. $56-71,2000$.

[8] C. Griffin and S. Duck, "Interferometric radio-frequency emitter location," IEE Proceedings - Radar, Sonar and Navigation, vol. 149, no. 3, p. $153,2002$.

[9] A. Amar and A. Weiss, "Localization of narrowband radio emitters based on doppler frequency shifts," IEEE Transactions on Signal Processing, vol. 56, no. 11, pp. 5500-5508, Nov. 2008. 
[10] J. Bhatti, "Sensor deception detection and radio-frequency emitter localization," Ph.D. dissertation, The University of Texas at Austin, Aug. 2015.

[11] W. Smith and P. Steffes, "Time delay techniques for satellite interference location system," IEEE Transactions on Aerospace and Electronic Systems, vol. 25, no. 2, pp. 224-231, Mar. 1989.

[12] K. Ho and Y. Chan, "Solution and performance analysis of geolocation by TDOA," IEEE Transactions on Aerospace and Electronic Systems, vol. 29, no. 4, pp. 1311-1322, 1993.

[13] D. Haworth, N. Smith, R. Bardelli, and T. Clement, "Interference localization for EUTELSAT satellites-the first European transmitter location system," International journal of satellite communications, vol. 15, no. 4, pp. 155-183, 1997.

[14] A. Kalantari, S. Maleki, S. Chatzinotas, and B. Ottersten, "Frequency of arrival-based interference localization using a single satellite," in 2016 8th Advanced Satellite Multimedia Systems Conference and the 14th Signal Processing for Space Communications Workshop (ASMS/SPSC). IEEE, Sept. 2016.

[15] M. J. Murrian, L. Narula, and T. E. Humphreys, "Characterizing terrestrial GNSS interference from low earth orbit," in Proceedings of the ION GNSS+ Meeting. Institute of Navigation, Oct. 2019.

[16] K. Becker, "An efficient method of passive emitter location," IEEE Transactions on Aerospace and Electronic Systems, vol. 28, no. 4, pp. 1091-1104, 1992.

[17] P. Ellis, D. V. Rheeden, and F. Dowla, "Use of doppler and doppler rate for RF geolocation using a single LEO satellite," IEEE Access, vol. 8, pp. 12907-12920, 2020.

[18] R. G. Brown and P. Y. Hwang, Introduction to Random Signals and Applied Kalman Filtering. Wiley, 2012.

[19] J. L. Crassidis and J. L. Junkins, Optimal estimation of dynamic systems. Chapman and Hall/CRC, 2011.

[20] T. E. Humphreys, M. J. Murrian, and L. Narula, "Deep-urban unaided precise global navigation satellite system vehicle positioning," IEEE Intelligent Transportation Systems Magazine, vol. 12, no. 3, pp. 109122,2020

[21] T. E. Humphreys, Interference. Springer International Publishing, 2017, pp. $469-503$.

[22] M. L. Psiaki and T. E. Humphreys, Position, Navigation, and Timing Technologies in the 21st Century: Integrated Satellite Navigation, Sensor Systems, and Civil Applications. Wiley-IEEE, 2020, vol. 1, ch. Civilian GNSS Spoofing, Detection, and Recovery, pp. 655-680.

[23] P. Welch, "The use of fast fourier transform for the estimation of power spectra: A method based on time averaging over short, modified periodograms," IEEE Transactions on Audio and Electroacoustics, vol. 15, no. 2, pp. 70-73, June 1967.

[24] T. E. Humphreys, M. L. Psiaki, and P. M. Kintner, "Modeling the effects of ionospheric scintillation on GPS carrier phase tracking," IEEE Transactions on Aerospace and Electronic Systems, vol. 46, no. 4, pp. 1624-1637, Oct. 2010.

[25] J. A. Barnes, Tables of bias functions, $B_{1}$ and $B_{2}$, for variances based on finite samples of processes with power law spectral densities. National Bureau of Standards, 1969.

[26] T. Bagala, A. Fibich, P. Kubinec, and V. Stofanik, "Improvement of short-term frequency stability of the chip scale atomic clock," in 2016 IEEE International Frequency Control Symposium (IFCS). IEEE, may 2016.

[27] United States Coast Guard, "GPS problem reports status," https://navcen. uscg.gov/?Do=gpsreportstatus, accessed 2020-08-31.

[28] P. Madhani, P. Axelrad, K. Krumvieda, and J. Thomas, "Application of successive interference cancellation to the GPS pseudolite near-far problem," IEEE Transactions on Aerospace and Electronic Systems, vol. 39, no. 2, pp. 481-488, April 2003. 